\title{
Early Identification of Foot and Lower Limb Stress Fractures using Diagnostic Ultrasonography: A review of three cases
}

\author{
by Sara L Jones $\mathrm{PhD}^{1 \rrbracket}$, Maureen Phillips $\mathrm{MSc}^{2 \underline{ }}$
}

The Foot and Ankle Online Journal 3 (4): 3

Early identification of the presence of stress fractures of the foot is often dependent upon clinical history and pattern of symptoms. The delay between presentation with symptoms and the confirmation of positive findings using plain film radiograph, or the invasive and more complex nature of bone scans or magnetic resonance imaging (MRI) can act as a deterrent to investigation. In contrast, the use of diagnostic ultrasonography in early detection of stress fractures of the foot and lower limb may offer a rapid and non-invasive technique for assessment and identification.

Key words: Diagnostic ultrasonography, Stress fracture.

Accepted: March, $2010 \quad$ Published: April, 2010

This is an Open Access article distributed under the terms of the Creative Commons Attribution License. It permits unrestricted use, distribution, and reproduction in any medium, provided the original work is properly cited. (The Foot and Ankle Online Journal (www.faoj.org)

$\mathrm{T}$ The treatment of stress fractures within clinical practice typically features a symptomatic approach to diagnosis. A history of high levels of activity and pain in a specific site are hallmarks of the likely presence of this pathology. Differential diagnosis may include contusion, Morton's neuroma and bone cyst or tumour.

Plain film radiographs involving stress fractures commonly show sclerosis and/or periosteal reaction approximately six weeks after the initial onset of pain. Evidence of fracture may not show up on radiograph for up to ten weeks. ${ }^{1,2}$

Address correspondence to : School of Health Sciences, University of South Australia, GPO Box 2471, Adelaide South Australia 5001.

E-mail: sara.jones@unisa.edu.au, maureen.phillips@unisa.edu.au

${ }^{1,2}$ School of Health Sciences, University of South Australia, GPO Box 2471, Adelaide South Australia 5001 .
By the time the diagnosis may be confirmed in this manner, many patients have become asymptomatic, or (of more concern) gone on to develop chronic symptoms. In most cases, earlier diagnosis will require more complex and invasive interventions, such as magnetic resonance imaging (MRI) or bone scans.

The literature shows that there has been extensive research into the use of diagnostic ultrasonography in investigation of soft tissue lesions ${ }^{3,4}$, but there appears to be little evidence of its use in the detection of stress fractures within the foot or lower limbs. The use of ultrasound in the diagnosis of stress fracture has been reported, but certainly not frequently. ${ }^{5}$ Case reports provide evidence that ultrasound imaging can be used in the detection of occult fractures. ${ }^{6}$ There are reports in the literature of the use of diagnostic ultrasonography to detect subperiosteal haematoma, periosteal elevation and metaphyseal injuries. ${ }^{7}$ 
Another study ${ }^{8}$ reported the use of ultrasound in imaging forearm fractures in children and found that ultrasound imaging appeared to be a viable alternative to radiography in simple fractures. More recently, Banal, et al., reported a case of stress fracture of the second metatarsal which was diagnosed by diagnostic ultrasound. They discussed that ultrasound investigation for stress fracture should be studied further because its non-invasiveness, low cost and ease of access could allow it to become the preferred method of diagnosis. Therapeutic ultrasound, with its potential to cause pain at a fracture site has proved both undesirable and also unreliable in detection of fractures. ${ }^{10,12}$

Imaging with ultrasound should yield good results. Bone is a natural obstacle to the transmission of sound at high frequency. ${ }^{8}$ There is a large difference in acoustic impedance between the soft tissue and bone resulting in a strong reflection from the bone. However, this presents an ideal situation for imaging the bony contour. Any imperfections (for example, steps, breaks, periosteal reactions) should therefore be easily visible. ${ }^{11}$ In cases where there is doubt as to the aetiology of the pain, and a need for differentiation of bony from soft tissue injury, diagnostic ultrasound can provide a good alternative to more invasive techniques such as X-ray or nuclear medicine.

As an added benefit in clinical practice, diagnostic ultrasound has the capability of demonstrating increased blood flow in periosteal reactions with the use of Doppler measures. ${ }^{11}$ We present a series of stress fracture cases which were diagnosed using diagnostic ultrasound. In each case, sonography was performed using a Siemen's Antares Sonoline ultrasound machine (Siemen's Medical Solutions, USA Inc, Ultrasound group, Issaquah, WA). A 13-5 $\mathrm{MHz}$ linear array transducer was used, with the frequency generally used between $10 \mathrm{MHz}$ and 11.5 $\mathrm{MHz}$. All subjects gave consent for use of the material relating to their case.

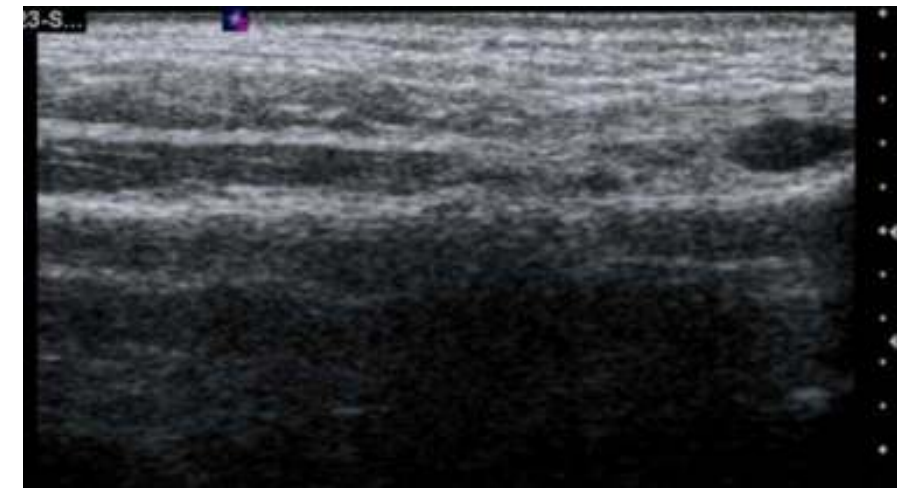

Figure 1 Periosteal thickening on ultrasound.

\section{Case 1: Tibial stress fracture}

A 22 year-old male athlete presented to the podiatry clinic complaining of a six week history of anterior tibial pain, right leg only, with worsening pain. Originally self-diagnosed (and self treated) as a soft tissue injury, treatment comprised icing, NSAIDs, changes in footwear and modifications in activity levels (but not complete rest).

On examination, the region of pain was localised. No evidence of swelling or bruising was noted. The area was tender to direct pressure. A stress fracture was suspected, but the patient was unconvinced. A diagnostic ultrasound scan was performed, as a noninvasive, low-radiation alternative to plain films or bone scan in order to more definitively identify the cause. Periosteal thickening was observed at the point of maximal tenderness on the anterior tibia, with underlying cortical irregularity. (Fig. 1) Power Doppler was negative, however, this was not considered unusual at six weeks post onset.

In this instance, identification of the presence of stress fracture by ultrasound and subsequent confirmation through bone scan (Fig. 2) resulted in a significant change in activity levels on the part of the athlete, with greater adherence to the recommended management regime. As a consequence, the injury fully resolved and there has been no recurrence to date. 


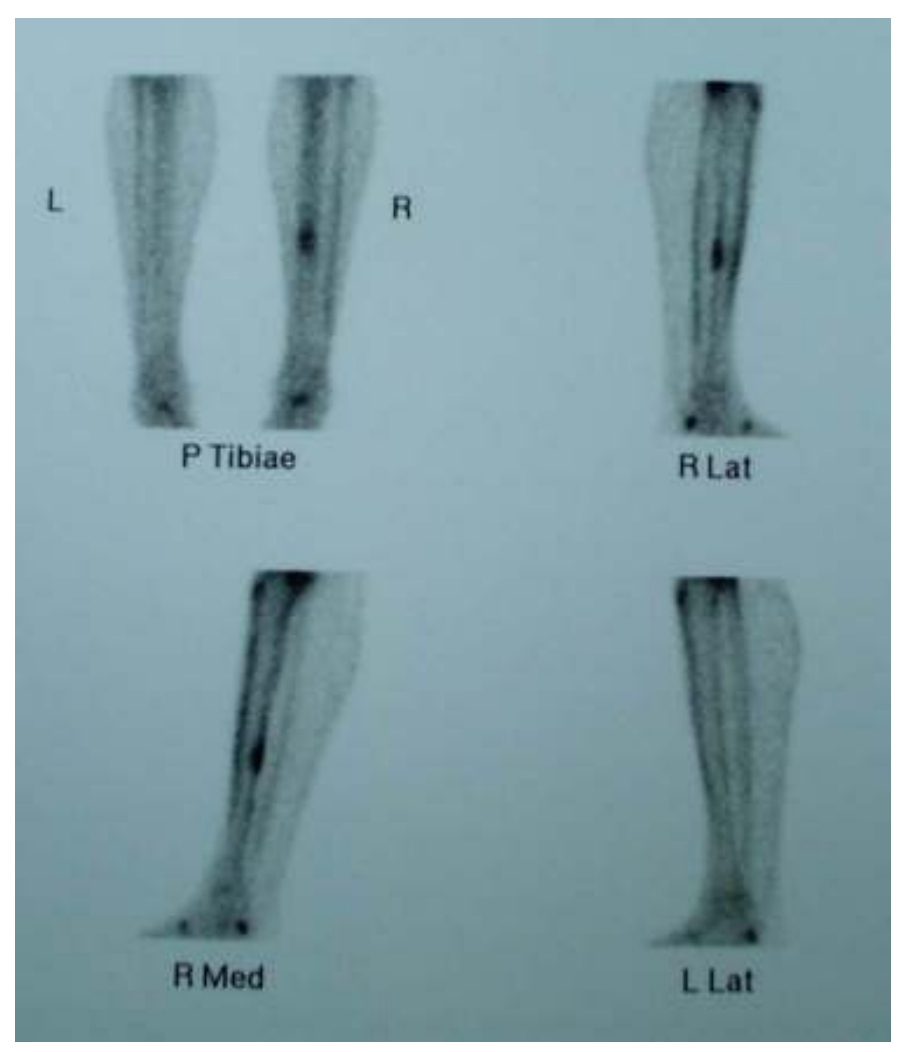

Figure 2 Bone scan findings, clearly showing an increase in uptake levels at the site of the stress fracture.

\section{Case 2: Metatarsal Stress Fracture}

A 16 year-old female presented with a five week history of foot pain, localised to the midfoot region, particularly around the third and fourth metatarsal shafts. Despite reporting pain, the patient had not altered her activity levels, which included netball, tennis and rock climbing. She reported a variable pattern of pain intensity, with reduction during periods of rest and exacerbation following activity. She sought treatment at the point when increased pain was experienced during as well as following activity, with an extended duration of symptoms. Previous radiograph examination had proved negative. The ultrasound findings show marked periosteal reaction with a strongly positive power doppler signal.
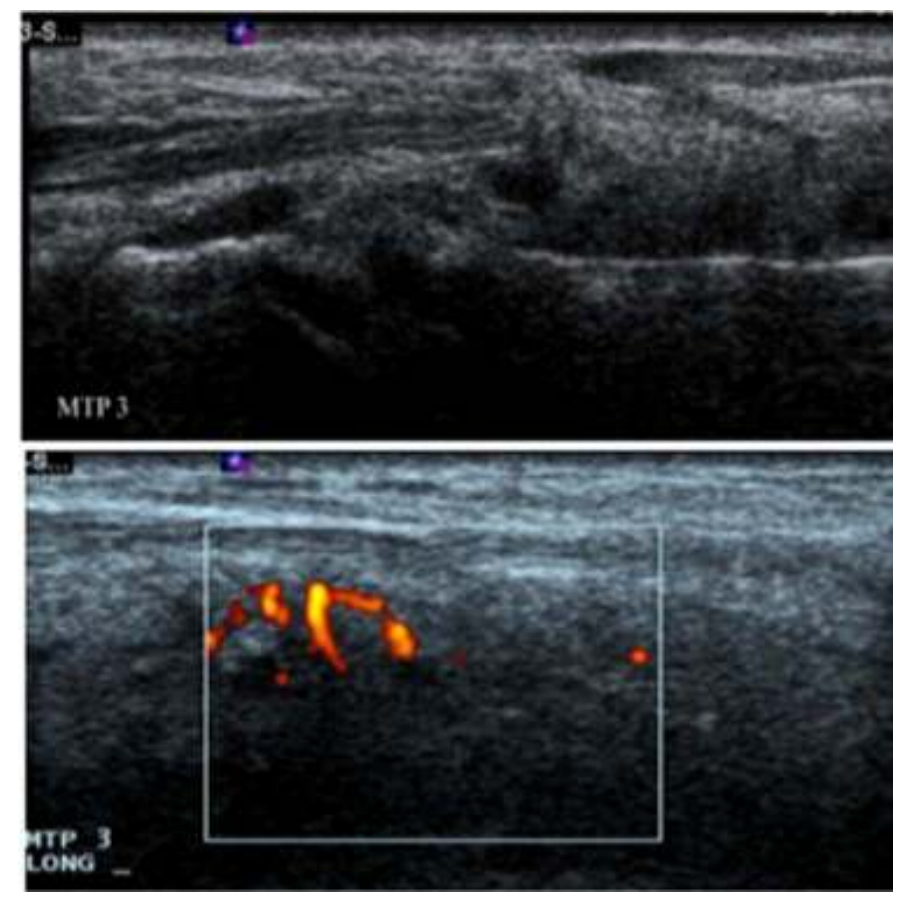

Figure 3 Diagnostic ultrasound findings. (Note the periosteal reaction)

A cortical fracture with callus formation is evident. (Fig. 3) Plain film radiographs were taken four weeks following the ultrasound examination. The films clearly show callus formation and confirmed the earlier diagnosis. (Fig 4)

Management in this case comprised a combination of activity modification, footwear changes and the use of orthoses, with gradual resolution of all symptoms and return to full sporting activity.

\section{Case 3: Metatarsal Head Fracture}

A 21 year-old female distance runner presented with a five day history of increasing pain under the fifth metatarsal head region during training. No change to footwear or activity levels in the period leading up to the onset of pain was reported. There was no history of trauma to the area. On examination, a clearly demarcated area of redness was visible in the area of the reported pain, which appeared to be consistent with a soft tissue, rather than bony injury. 


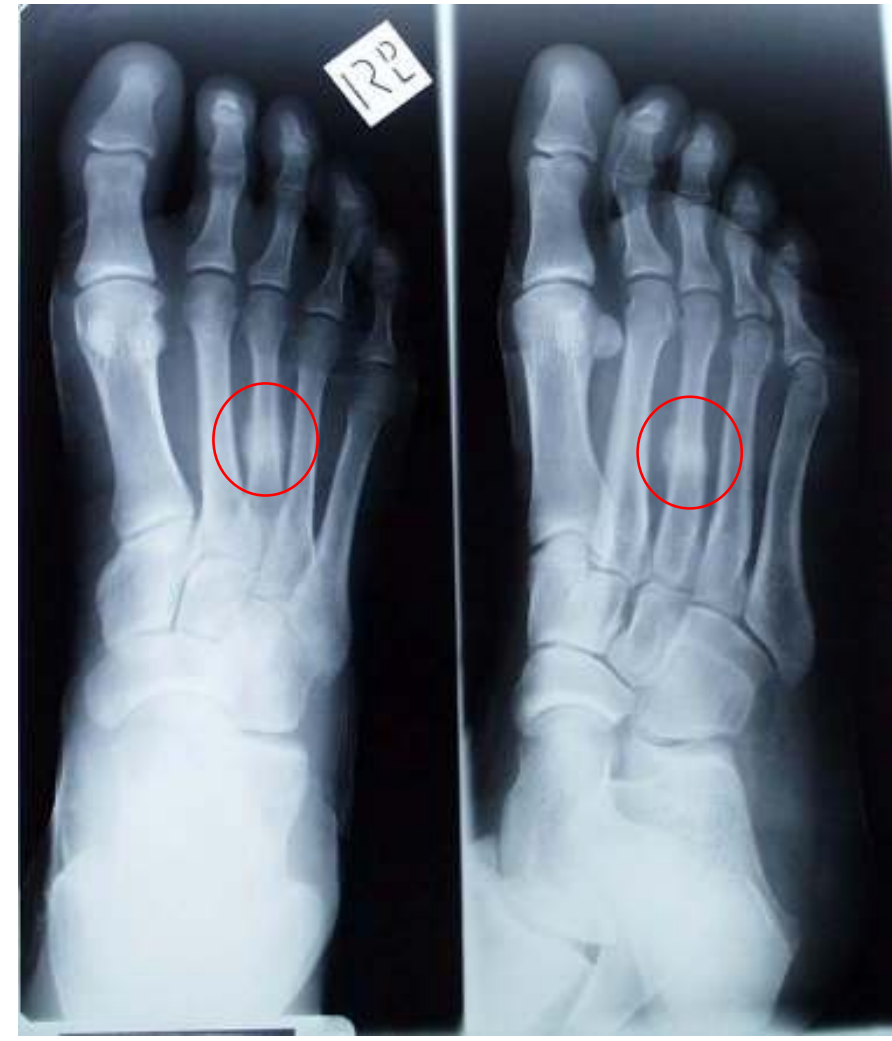

Figure 4 Plain film views showing the site of the stress fracture on the third metatarsal.

However, the reported symptoms and pattern of pain were not consistent with this diagnosis and a diagnostic ultrasound was therefore performed. (Fig 5) A clear break can be seen in the cortical bone (arrowed) with accompanying periosteal reaction and a positive power doppler signal. (Fig 6)

As a consequence, the injury was treated as a stress fracture, with cessation of the sporting activities and footwear management. The injury resolved over a six week period, with a return to competitive running at the end of this time without further symptoms. The area of inflammation noted on initial examination completely disappeared within two days of the initial consultation.

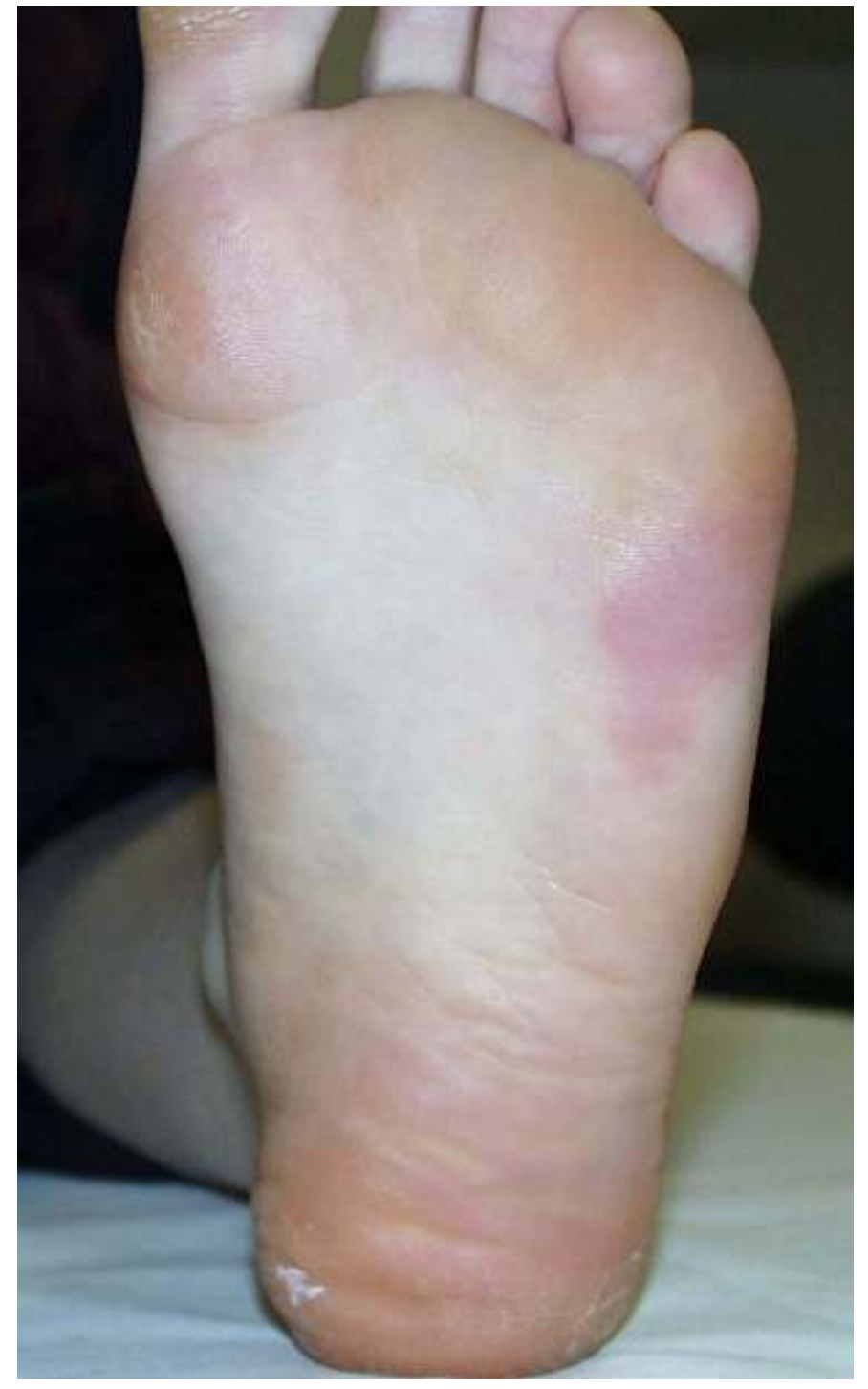

Figure 5 Clinical presentation. (Note the area of redness)

\section{Discussion}

Ultrasound has been previously recognised as a valuable tool in the identification of soft tissue pathologies affecting the foot, notably in the detection of tissue trauma, neuroma and other intermetatarsal masses. ${ }^{12}$ There is less evidence of its use as a means to identify bony pathology. 


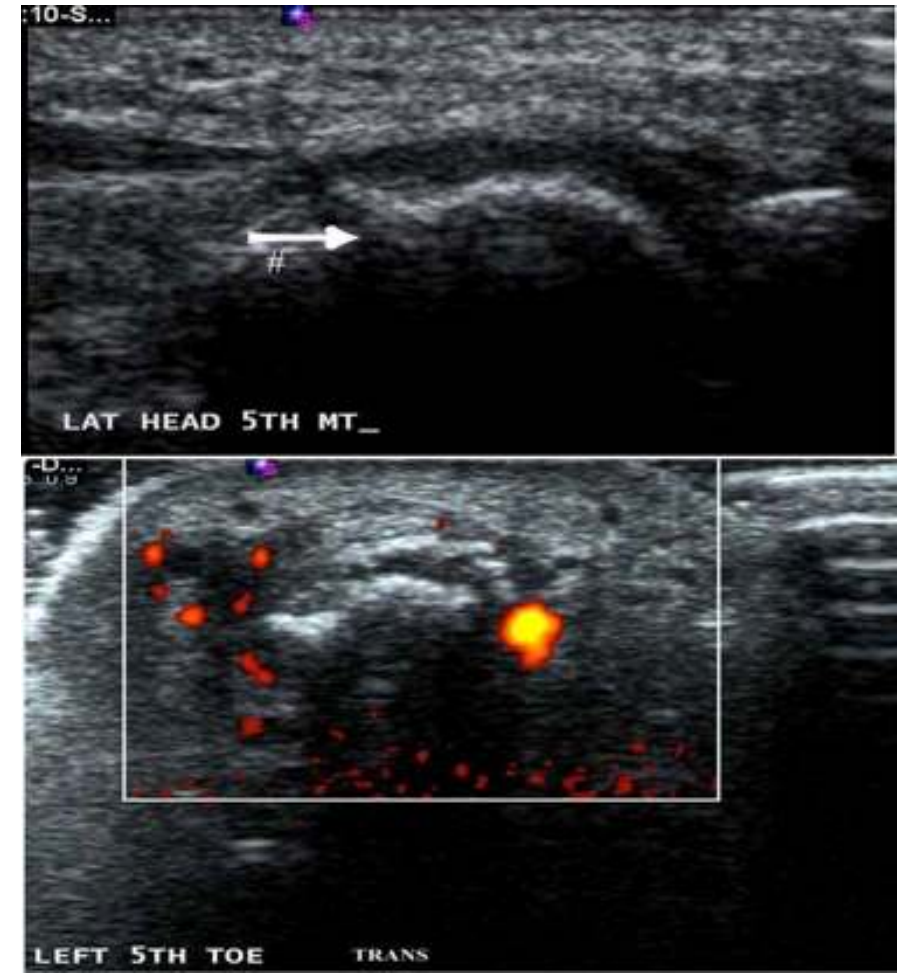

Figure 6 Site of stress fracture on ultrasound.

Previous trials using ultrasound to detect fractures have used therapeutic rather than imaging frequencies, that is $1 \mathrm{MHz}$ rather than $7.5 \mathrm{MHz}$ or greater. Therapeutic wavelengths often - but not consistently elicit pain at a fracture site ${ }^{13,14}$, which is often perceived as a disincentive for the use of ultrasound in this setting. Pain with the use of therapeutic ultrasound is caused by the vibrations from the transducer head. However, there were no reports of pain or discomfort from patients while using high resolution linear probe over the suspected fracture sites, suggesting that this should not be an issue in undertaking the diagnostic procedure.

The significance of the utilization of diagnostic ultrasound is in its ability to identify and localize the presence of stress fractures much earlier than other modalities. In Case 3, this identification was possible within days of onset of pain, allowing clear identification to the patient of the causes and the necessary management strategy.
This provision of clear evidence assisted greatly in patient compliance to the recommended management strategy by dispelling any belief that it was a soft tissue injury that could be "run out".

The increasing availability of office-based diagnostic ultrasound units for medical and allied health practices in recent years means that ready access to this technology may assist practitioners to provide earlier identification and implementation of treatment.

\section{Conclusion}

Use of diagnostic ultrasound in the diagnosis of stress fracture allows for an early diagnosis using a low cost, non-invasive modality. Its use in identification of both soft tissue and bony injury makes it a useful tool in diagnosis and subsequent management. The lack of reported clinical studies focusing upon the reliability and application of this technique in comparison with plain film and MRI suggests that it may be an area worthy of further investigation.

\section{References}

1. Monteleone GP Jr. Stress fractures in the athlete. Orthop Clin North Am 199526 (3): 423-432.

2. Brukner P. Exercise- related lower leg pain: bone. Med Sci Sports Exerc 200032 (3 suppl): S15-26.

3.Sofka C. Ultrasound in Sports Medicine. Sem Musculoskeletal Radiol 2004 8: 17-27.

4. Balint GP, Korda J, Hangody L, Balint P. Foot and ankle disorders. Best Prac Res Clin Rheum 200317 (1): 87-111.

5. Cho K-H, Lee Y-H, Lee S-M, Shahid MU, Suh KJ, Choi JH. Sonography of bone and bone related diseases of the extremities. J Clin Ultrasound 2004 32: 511-521.

6. Hunter JD, Mann CJ, Hughes PM. Fibular fracture: detection with high-resolution diagnostic ultrasound. J Accid Emerg Med 1998 15: 118-124.

7. Gleeson AP, Stuart MJ, Wilson B, Phillips B. Ultrasound assessment and conservative management of inversion injuries of the ankle in children. JBJS 1996 78B (3): 484-487.

(C) The Foot and Ankle Online Journal, 2010 
8. Williamson D, Watura R, Cobby M. Ultrasound imaging of forearm fractures in children: a viable alternative? J Accid Emerg Med 2000 17: 22-24.

9. Banal F, Etchepare F, Rouhier B, C Rosenberg, V Foltz, S Rozenberg. Ultrasound ability in early diagnosis of stress fracture of metatarsal bone. Annals Rheum Diseases 2006, 65: 977-978.

10. Shenouda NA, England JP. Ultrasound diagnosis of scaphoid fractures. J Hand Surg Br 1987 12: 43-45.

11. Van Holsbeeck MT, Introcaso JH. Musculoskeletal Ultrasound. Second edition. St. Louis Mosby. 2001.

12 Oliver $\mathrm{T}$, Beggs I. Ultrasound in the assessment of metatarsalgia: a surgical and historical correlation. Clin Radiology 1998 53: 287 -289.

13. DaCruz DJ, Taylor RH, Savage B, Bodiwala G. Ultrasound assessment of suspected scaphoid fracture. Arch Emerg Med 1988 5: 97-100.

14. Moss A, Mowat AG. Ultrasonic assessment of stress

fractures. BMJ 1983 286: 1949. 